\title{
LES GLACIERS ANDINS : UNE MÉMOIRE DU CLIMAT TROPICAL
}

\author{
Françoise VIMEUX ${ }^{1}$
}

\begin{abstract}
RÉSUMÉ
Reconstruire les variations passées de notre climat permet de mieux appréhender le fonctionnement de notre système climatique. Les carottes de glace issues des calottes polaires et des glaciers montagneux de haute altitude sont de très bonnes archives climatiques. Les glaciers présents sur les sommets des Andes sud-américaines sont ainsi exploités depuis les années 80. Une dizaine de carottages ont été réalisés depuis l'équateur jusqu'en Patagonie. Ils recouvrent des périodes très différentes allant du dernier siècle, jusqu'à la dernière transition glaciaire-interglaciaire qui débute il y a 21000 ans. La datation de ces carottes combine un ensemble de méthodes : comptage des cycles saisonniers des éléments chimiques et de la composition isotopique de la glace, analyse de la concentration des éléments radioactifs issus des tests atmosphériques nucléaires au xxe siècle et modélisation de l'écoulement du glacier pour la partie la plus profonde. Le présent article illustre deux résultats intéressants apportés par l'étude de la composition isotopique de ces glaces. Le premier concerne la période du Petit Âge Glaciaire au XVII-XVIII' siècle. L'analyse isotopique de la glace de deux carottes prélevées en Bolivie (Illimani) et au Pérou (Quelccaya), couplée à l'étude de l'extension des glaciers au même moment, montre des conditions climatiques légèrement plus humides et plus froides de 1650 à 1780 . Le second résultat est déduit de l'analyse isotopique d'une carotte prélevée en Patagonie du Nord (San Valentin) qui recouvre les 120 dernières années. L'enregistrement isotopique suggère que la température dans cette région a progressivement diminué au cours de cette période d'environ $0,2^{\circ} \mathrm{C}$ par décennie, venant prolonger une tendance mesurée par les stations météorologiques chiliennes depuis les années 70 malgré un contexte de réchauffement global.
\end{abstract}

Mots clés : carotte de glace, Andes, isotopes de l'eau, datation, Petit Âge Glaciaire, changement climatique

\section{ABSTRACT}

\section{ANDEAN GLACIERS: MEMORY OF TROPICAL CLIMATE}

The reconstruction of past climate variability offers important information about our climate system. Ice cores extracted from polar ice sheets or from mountainous glaciers are among the most popular climate archives. Ice cores from Andean glaciers in South America have been studied since the 1980's. Almost ten ice cores have been extracted from the equator to Patagonia. They cover different time periods from the last century to the last glacial-interglacial termination that started 21,000 years ago. Those ice cores are usually dated by using a combination of methods: annual layers counting in both isotopic and chemical ice composition, radionuclides concentration analysis to detect atmospheric nuclear tests during the $20^{\text {th }}$ century and ice flow modeling for the deepes part of those cores. This paper presents two interesting results arising from the isotopic composition of the ice. The first one deals with the isotopic records of Bolivian (Illimani) and Peruvian (Quelccaya) ice cores in regards to the reconstruction of glacier extent during the little ice age in the $17^{\text {th }}-18^{\text {th }}$ century. Both approaches suggest a slightly moister and cooler period from 1650 to 1780 . The second result arises from the 120-year long isotopic record of an ice core drilled in Patagonia (San Valentin). It suggests that at the regional scale, temperature has regularly decreased of about $0.2^{\circ} \mathrm{C}$ per decade, extending the temperature decrease shown by Chilean meteorological data since the 1970 's despite a global warming.

Keywords: ice core, Andes, water stable isotopes, dating, little ice age, climate change

\section{1 - POURQUOI ET COMMENT RECONSTRUIRE L'ÉVOLUTION PASSÉE DE NOTRE CLIMAT?}

Anticiper l'évolution à venir de notre climat est un enjeu majeur pour définir les politiques futures d'adaptation et de mitigation. Ce défi ne peut être relevé que si nous avons une très bonne connaissance des rouages $\mathrm{du}$ système climatique, de ses modes de variabilité et de leurs déclinaisons à l'échelle régionale. Regarder comment le climat a varié dans le passé est une manière d'aborder ces questions brûlantes. Tout système naturel qui a conservé dans sa structure ou dans sa composition chimique ou isotopique des traces des variations climatiques passées est appelé « archive climatique ».

Parmi les archives les plus étudiées, on compte par exemple les sédiments marins et lacustres, les arbres qui forment un cerne annuel, les spéléothèmes qui croissent en formant des lamines calcaires au fur et à mesure que l'eau de pluie s'écoule dans les grottes, ou encore les glaciers et les calottes polaires formées par les neiges qui s'accumulent année après année et gardent en mémoire les conditions climatiques de l'époque de leur formation. 
L'étude de ces archives consiste à traduire les informations qu'elles recèlent en termes de variables climatiques quantifiées (température et quantité de pluie sont les reconstructions paléo-climatiques les plus fréquentes).

La glace est une précieuse archive climatique. Depuis près de cinquante ans, les glaces du Groenland et de l'Antarctique sont exploitées pour reconstruire l'histoire de notre climat à l'échelle globale sur des périodes couvrant jusqu'à 800000 ans environ (EPICA community members, 2004). Dans les années 80 , les scientifiques se sont interrogés sur le potentiel des carottes de glace extraites des glaciers de haute montagne en Amérique du Sud pour étudier le climat de ce continent (Garreaud et al., 2009).

\section{2 - LES CAROTTES DE GLACE ANDINES, DES FORAGES DES ANNÉES 1980 À AUJOURD'HUI}

Une équipe américaine menée par Lonnie Thompson (professeur à l'université de l'État d'Ohio aux ÉtatsUnis) a prélevé pour la première fois, en 1983, une carotte de glace dans un glacier de haute altitude dans les Andes péruviennes (Quelccaya, $5670 \mathrm{~m}, 13^{\circ} 56^{\prime} \mathrm{S}$, $\left.70^{\circ} 50^{\prime} \mathrm{W}\right)$. Ce forage faisait suite à une première reconnaissance sur place en 1974 concluant à une excellente conservation des couches de neige annuelles (cet aspect est primordial pour fournir des enregistrements de qualité, non altérés par des fontes successives de la glace en été). L'analyse de deux carottes d'une longueur de près de $160 \mathrm{~m}$ a montré que ce glacier renfermait des indices climatiques inédits. Ce sont 1500 années d'histoire du climat qui ont pu être reconstituées, avec une résolution saisonnière sur la majeure partie de l'enregistrement (Thompson et al., 1985). Face à la richesse des informations climatiques potentiellement préservées dans ces glaciers sud-américains, d'autres forages dans les Andes tropicales ont été initiés par la suite (fig. 1). En 1993, un forage au sommet du Huascarán (Pérou, $6050 \mathrm{~m}, 9^{\circ} 06^{\prime} \mathrm{S}$, $77^{\circ} 30^{\prime} \mathrm{W}$ ) a offert pour la première fois la possibilité de reconstruire les variations climatiques tropicales sur près de 18000 ans, englobant ainsi la dernière déglaciation (Thompson et al., 1995). Les carottages des glaciers sommitaux du Sajama en 1997 (Bolivie, 6542 m, cordillère occidentale, $\left.18^{\circ} 06^{\prime} \mathrm{S}, 68^{\circ} 53^{\prime} \mathrm{W}\right)$, de l'Illimani en 1999 (Bolivie, $6350 \mathrm{~m}$, cordillère orientale, 16 $6^{\circ} 37^{\prime} \mathrm{S}$, $67^{\circ} 46^{\prime} \mathrm{W}$ ), du Chimborazo en 2000 (Equateur, $6270 \mathrm{~m}$, $1^{\circ} 28^{\prime} \mathrm{S}, 78^{\circ} 48^{\prime} \mathrm{W}$ ) et du Coropuna en 2003 (Pérou, $\left.6425 \mathrm{~m}, 15^{\circ} 32^{\prime} \mathrm{S}, 72^{\circ} 39^{\prime} \mathrm{W}\right)$, réalisés par l'Institut de Recherche pour le Développement (IRD) et ses partenaires sud-américains et européens, sont venus compléter le panorama climatique de cette région du monde sur des périodes remontant jusqu'à 25000 ans avant aujourd'hui (Thompson et al., 1998; Ramirez et al., 2003). En 2007, ces mêmes équipes se sont intéressées à des sites extratropicaux et ont extrait une carotte de glace au sommet du Mont San Valentin sur la calotte nord Patagonienne (Chili, $3747 \mathrm{~m}, 46^{\circ} 35^{\prime} \mathrm{S}, 73^{\circ} 19^{\prime} \mathrm{W}$ ). Les résultats obtenus sur les 140 dernières années ont permis d'explorer la variabilité climatique de cette région, pauvre en données météorologiques avant les années 1960, et sa réponse aux prémices du changement climatique actuel (Vimeux et al., 2008).

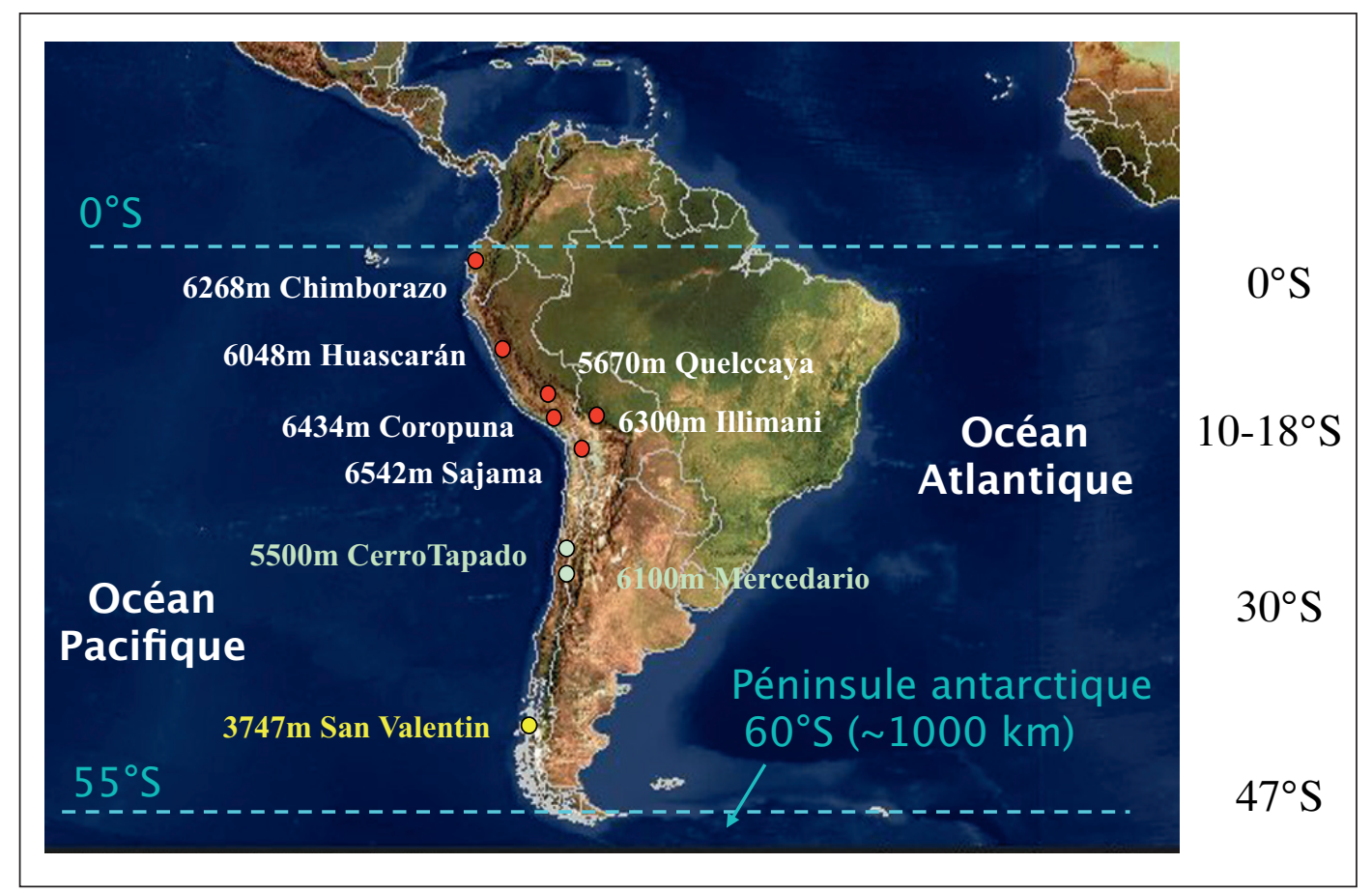

Fig. 1 : Localisation des sites de forages dans les Andes, depuis l'équateur jusqu'à La Patagonie.

Les sites de forages sont indiqués par des cercles (rouge: région tropicale, vert: région subtropicale, jaune: région de moyenne latitude) avec leur altitude. Fig. 1: Location of drilling sites in the Andes from the equator to Patagonia. Drilling sites are indicated by circles (red for tropical regions, green for subtropical regions and yellow for mid-latitudes) with their altitude. 


\section{3 - QUELLES INFORMATIONS CLIMATIQUES PEUT-ON TROUVER DANS LES CAROTTES ANDINES ET COMMENT SONT-ELLES DATÉES ?}

Deux types de mesures sont généralement réalisées sur les carottes de glace andines : la composition isotopique de la glace (c'est-à-dire le rapport entre le nombre de molécules de type $\mathrm{HDO}$ ou $\mathrm{H}_{2} \mathrm{O}^{18}$ et la molécule $\mathrm{H}_{2} \mathrm{O}^{16}$ la plus abondante) et la concentration en éléments chimiques (calcium, sulfate, sodium, etc.). Elles sont souvent complétées par l'analyse de la concentration en radionucléides $\left({ }^{137} \mathrm{Cs},{ }^{241} \mathrm{Am},{ }^{210} \mathrm{~Pb}\right.$ par exemple) qui apporte des éléments précieux pour la datation de ces carottes sur la période récente (le $\mathrm{xx}^{\mathrm{e}}$ siècle.).

$\mathrm{Si}$ la composition isotopique de la glace polaire apporte une information en termes de température atmosphérique (Jouzel et al., 2003), il en est autrement sous les tropiques où le cycle de l'eau est beaucoup plus complexe. Tout d'abord, les pluies (et donc la neige) sont majoritairement issues de nuages convectifs dans lesquels les gouttelettes d'eau subissent des processus d'évaporation modifiant à chaque fois leur composition isotopique. De plus, les masses d'air tropicales sont en partie recyclées via la végétation (transpiration) et mélangées avec des sources extérieures telles que l'évaporation de l'eau de pluie interceptée par la canopée et des eaux stagnantes (rivière, zone inondée) qui modifient fortement leur composition isotopique. Il est ainsi plus difficile d'exploiter les profils isotopiques des carottes de glace tropicales en termes d'une seule variable climatique quantifiée. Depuis une quinzaine d'années, de gros efforts couplant des observations isotopiques directes sur les pluies et la modélisation du cycle atmosphérique des isotopes de l'eau ont permis de montrer que la composition isotopique des neiges andines répond principalement à l'intensité des pluies sud-américaine lors de la saison de mousson (la mousson sud-américaine correspond à la saison des pluies par analogie avec la mousson asiatique et africaine ; au cours de cette saison, de fortes pluies se produisent grâce à l'apport d'humidité depuis l'Océan Atlantique). Celle-ci se développe de novembre à mars et apporte environ $70 \%$ des neiges tombées sur la chaîne andine. Pour expliquer cette relation intime entre les isotopes de l'eau et l'intensité des pluies de mousson, partons de la source des précipitations. En s'évaporant, la surface de l'océan crée une vapeur d'eau légèrement enrichie en molécules d'eau légères $\left(\mathrm{H}_{2} \mathrm{O}^{16}\right)$ par rapport aux molécules lourdes ( $\mathrm{HDO}$ ou $\mathrm{H}_{2} \mathrm{O}^{18}$ ) qui restent préférentiellement dans la phase condensée. Cet appauvrissement de la vapeur formée en molécules lourdes, appelé fractionnement isotopique, est la conséquence d'une pression de vapeur saturante légèrement plus élevée pour les molécules de type $\mathrm{HDO}$ ou $\mathrm{H}_{2} \mathrm{O}^{18}$. Au cours de son voyage, cette masse d'air va se refroidir en s'élevant lors des ascendances liées à la convection, et par conséquent son contenu en vapeur d'eau va se condenser. A chaque condensation, la vapeur d'eau s'appauvrit encore un peu plus en molécules lourdes, qui quittent la masse d'air définitivement emportées par la précipitation (encore une fois, ce processus d'appauvrissement provient des différences de pression de vapeur saturante entre les différentes formes de la molécule d'eau). Ainsi comprend-on que plus les pluies sont intenses au cours du voyage de la masse d'air depuis sa source jusqu'aux sommets andins, plus les neiges précipitant sont appauvries en molécules lourdes. La composition isotopique des glaces andines permet donc de retracer l'histoire de la mousson sudaméricaine qui est un élément majeur du climat de cette région, et qui répond aux grands bouleversements climatiques globaux (Vimeux et al., 2005 ; 2011).

L'atmosphère ne transporte pas que des pluies, elle apporte aussi des poussières, du pollen, des cendres volcaniques, des sels marins, des résidus de feux de biomasse que l'on retrouve dans les glaciers par dépôt direct en surface ou via les dépôts neigeux. Ainsi, l'analyse chimique de ces divers composés nous délivre-t-elle des messages importants sur la circulation atmosphérique passée, l'intensité des vents mais aussi l'impact de l'homme sur son environnement (apport de composés issus de la pollution anthropique).

Enfin, l'analyse des traces des explosions nucléaires atmosphériques dans les années 60 et 70 dans l'hémisphère sud apporte des éléments de datation absolus et donc précieux (fig. 2). Ceux-ci, couplés à d'autres méthodes de datation usuelles (comptage des cycles saisonniers des espèces isotopiques et chimiques, modélisation de l'écoulement de la glace, mesures du rapport isotopique de l'oxygène des bulles d'air, mesures en carbone 14, etc.), permettent de proposer une échelle de temps pour l'ensemble des reconstructions. En règle générale, l'erreur sur la datation est faible (de quelques années) sur la période où il est possible de coupler le comptage des cycles saisonniers à des repères absolus comme les tests nucléaires.

\section{4 - QUELQUES RÉSULTATS MAJEURS, ET INATTENDUS !}

L'étude des climats tropicaux a été tardive. Jusqu'à il y encore une vingtaine d'années, la variabilité du climat tropical était plutôt perçue comme une réponse de faible amplitude aux variations climatiques qui naissaient et se développaient dans les plus hautes latitudes sous l'effet de forçages extérieurs naturels (ensoleillement, éruption volcanique, variations de l'orbite terrestre) ou anthropiques. La machine climatique est en réalité bien plus complexe. Les régions tropicales ont une dynamique propre face aux forçages et participent aux variations climatiques globales via des mécanismes de télé connexions atmosphériques ou océaniques et des rétroactions complexes qu'il est important de décortiquer. Il ne faut pas oublier, par exemple, que c'est dans le Pacifique tropical que prend naissance le premier mode de variabilité climatique interannuelle. Il s'agit du phénomène « El Niño » dont l'événement de 2015-2016 que nous venons de vivre a été particulièrement intense et a eu de fortes répercussions dans plusieurs régions du monde (voir par exemple: https://www.climate.gov/enso). 


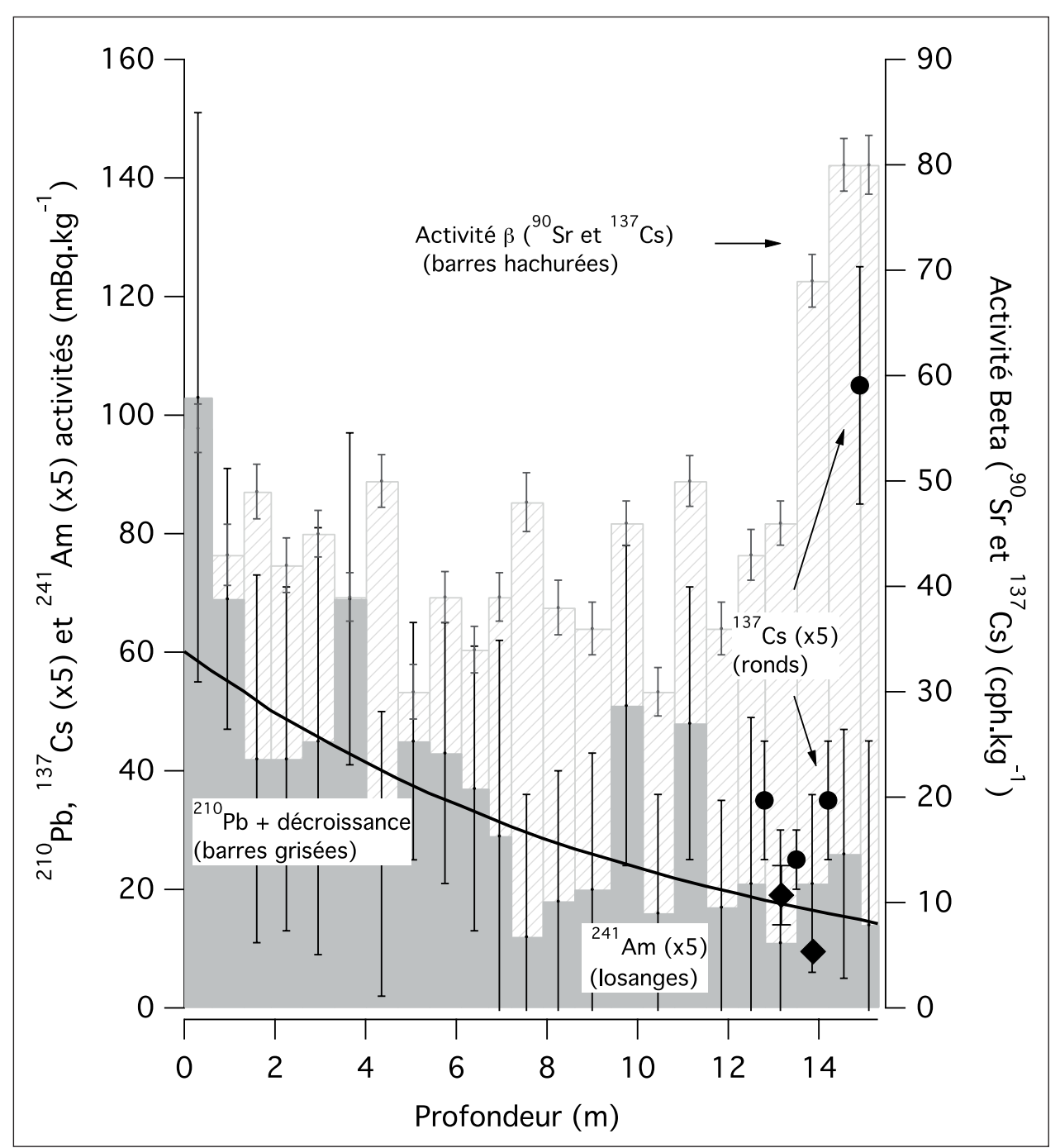

Fig. 2 : Enregistrement des radionucléides dans une carotte courte $(15 \mathrm{~m})$ prélevée au sommet du mont San Valentin en Patagonie.

Émission gamma, ${ }^{137} \mathrm{Cs},{ }^{210} \mathrm{~Pb},{ }^{241} \mathrm{Am}$ et émission beta ${ }^{90} \mathrm{Sr}$ plus ${ }^{137} \mathrm{Cs}$ ). Le profil de décroissance du ${ }^{210} \mathrm{~Pb}$ et la présence de ${ }^{137} \mathrm{Cs}$ et ${ }^{241} \mathrm{Am}$ au fond de cette carotte courte indiquent une date postérieure de 1 ou 2 ans aux essais nucléaires atmosphériques des années 1964-1965 pour le fond de cette carotte (Vimeux et al., 2008).

Fig. 2: Radionuclide profiles in a fern core $(15 \mathrm{~m})$ extracted from Monte San Valentin in Patagonia. Gamma emitters, ${ }^{137} \mathrm{Cs},{ }^{210} \mathrm{~Pb},{ }^{241} \mathrm{Am}$ as well as beta emitters ${ }^{90} \mathrm{Sr}$ plus $\left.{ }^{137} \mathrm{Cs}\right)$. The ${ }^{210} \mathrm{~Pb}$ decreasing trend and the high level of ${ }^{137} \mathrm{Cs}$ and ${ }^{241} \mathrm{Am}$ in the deepest part of the core suggest an age older by $1-2$ years than the nuclear atmospheric tests in 1964-1965 (Vimeux et al., 2008).

Les carottes de glace issues des glaciers andins sont donc une aubaine pour mieux comprendre le comportement du climat tropical au fil du temps, dans des contextes de forçages très différents. La suite de l'article illustre deux résultats qui sont issus de l'étude de ces carottes.

\section{1 - LE PETIT ÂGE GLACIAIRE DANS LES ANDES TROPICALES}

Le terme de «Petit Âge Glaciaire » (PAG) désigne une période relativement froide allant $d u \mathrm{Xv}^{\mathrm{e}}$ à $\mathrm{la}$ fin $\mathrm{du}$ $\mathrm{XIX}^{\mathrm{e}}$ siècle avec un paroxysme entre les années 1600 et 1800 environ, suivant les régions considérées. L'extension géographique de ce phénomène, tout comme son origine, sont débattues mais un consensus existe pour dire que ce phénomène a été centré sur l'Europe. Les conditions climatiques plus froides que de nos jours au cours de cette période ont été mises en évidence grâce à l'étude de plusieurs archives climatiques comme les cernes d'arbres mais aussi grâce à la lecture de nombreux écrits historiques, et au travers d'anciennes gravures et peintures qui l'attestent (voir les tableaux du peintre Pieter Bruegel laissant supposer des hivers particulièrement froids dans le nord de l'Europe à cette époque).

Dans l'hémisphère sud, les traces de cette période sont plus rares. En Amérique du Sud, des écrits historiques laissent à penser qu'une période similaire s'est produite avec toutefois des incertitudes sur sa datation. Avons-nous la trace d'un tel événement dans les carottes andines ? La très bonne datation des deux carottes forées sur les sommets des monts Illimani et Quelccaya (Ramirez et al., 2003 ; Thompson et al., 1985) permet de s'attarder sur cette question. On note sur les deux enregistrements isotopiques issus de ces carottes qu'à partir de 1650 la composition isotopique de la glace diminue (d'environ 1,5\% sur le rapport oxygène18/oxygène16) et ne revient à son niveau précédent que vers 1780 , ce qui pourrait s'interpréter comme une augmentation de la quantité de pluie en amont des Andes perdue par les 


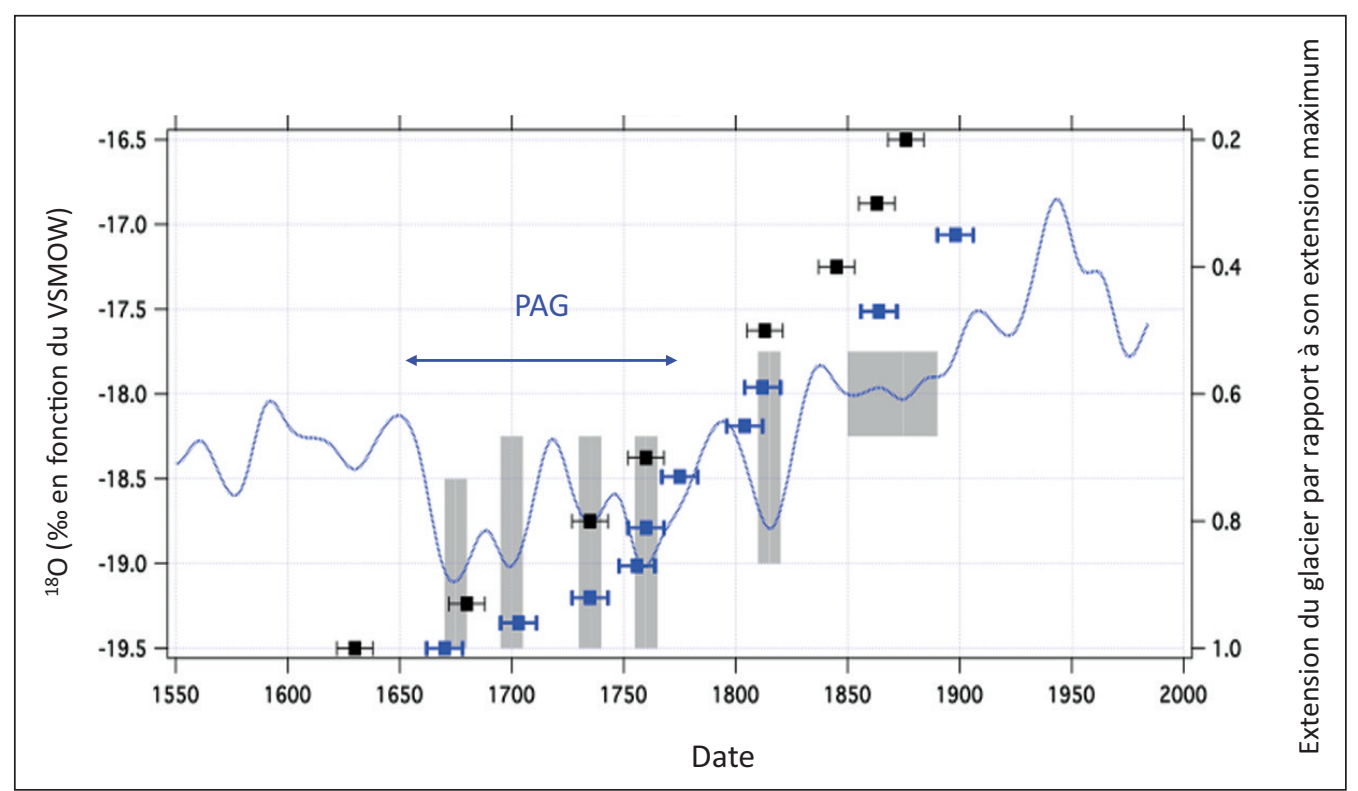

Fig. 3 : Composition isotopique moyenne en oxygène 18 des carottes de glace d'Illimani et de Quelccaya depuis 1550 et extension des glaciers en Bolivie et au Pérou.

La courbe isotopique est tracée en bleu. Les symboles carrés représentent les dates moyennes des avancées maximum successives de plusieurs glaciers en Bolivie (bleu) et au Pérou (noir) déterminées par l'étude de l'âge des moraines glaciaires (adapté de Jomelli et al., 2009). On constate une bonne cohérence entre des périodes d'avancées maximums de glaciers, et de plus fortes précipitations (rapport isotopique minimum).

Fig. 3: Mean isotopic composition (oxygen 18) of Illimani and Quelccaya ice cores since 1550 and glacial extents in Bolivia and Peru. The isotopic composition is in blue (\%o versus VSMOW). Periods of glacier maximal advances are represented by square symbols (blue for Bolivia and black for Peru). They are determined by moraine ages (from Jomelli et al., 2009). There is a consistency between glacial advances and high level of rain (isotopic minimum).

masses d'air le long de leur trajectoire, au-dessus du Brésil (fig. 3). Il n'y a pas d'indication en termes de température dans ces mesures mais il est avéré qu'à cette même époque, l'avancée des glaciers andins était maximale reflétant des conditions probablement plus froides, et éventuellement plus humides (Jomelli et al., 2009) (fig. 3). Ainsi, le PAG aurait-il aussi laissé des traces dans les Andes se déclinant comme étant une période plus froide mais aussi plus humide qu'aujourd'hui. Récemment, une étude paléoclimatique sur cette même période à partir de spéléothèmes brésiliens laminés annuellement est venue confirmer l'existence d'une période plus humide au Brésil durant le PAG (Novello et al., 2016).

\section{2 - UN REFROIDISSEMENT DANS LES ANDES CHILIENNES DANS UN MONDE QUI SE RÉ- CHAUFFE}

L'analyse de la carotte de glace forée au sommet du mont San Valentin en 2007 (d'une longueur de 122 m jusqu'au socle rocheux) a permis de recouvrir avec une résolution annuelle les derniers 140 ans. A cette latitude, la composition isotopique de la glace, comme dans les régions polaires, est majoritairement contrôlée par la température (Jouzel et al., 2003). Le profil de température reconstitué depuis 1880 environ montre une tendance générale au refroidissement, d'environ $0,2{ }^{\circ} \mathrm{C}$ tous les 10 ans. Un tel refroidissement a aussi été observé depuis 1979 sur la côte chilienne à partir des données météorologiques et des données satellites (Falvey \& Garreaud, 2006). L'enregistrement glaciaire chilien permet d'élargir les observations disponibles sur la période instrumentale, et de montrer que le refroidis- sement récent a en réalité débuté il y a plus de 140 ans. Des études s'appuyant sur la modélisation climatique montrent que le réchauffement global modifie la position et l'intensité de l'anticyclone présent dans le Pacifique Sud. En conséquence, cela favorise les advections d'air froid vers le continent depuis l'océan et les remontées d'eau froide le long de la côte chilienne (appelées « upwelling ») expliquant un léger refroidissement dans cette région du globe.

Les deux exemples que nous avons cités dans cette section montrent comment les réponses du système climatique aux forçages climatiques (un ensoleillement légèrement plus faible au cours du PAG ; une augmentation des gaz à effet de serre dans l'atmosphère depuis le début du XIX ${ }^{\mathrm{e}}$ siècle) sont enregistrées dans l'archive glaciaire. Ils montrent aussi la nécessité de s'attarder sur les réponses régionales à un changement climatique global (qu'il soit d'origine anthropique ou naturelle) si l'on souhaite émettre des politiques d'adaptation fiables à ces échelles.

\section{RÉFÉRENCES BIBLIOGRAPHIQUES}

EPICA COMMUNITY MEMBERS, 2004 - Eight glacial cycles from an Antarctic ice core. Nature, 429, 623-628.

FALVEY M. \& GARREAUD R.D., 2009 - Regional cooling in a warming world: Recent temperature trends in the southeast Pacific and along the west coast of subtropical South America (1979-2006). Journal of Geophysical Research, 114, D04102, doi:10.1029/2008JD010519.

GARREAUD R.D., VUILLE M., COMPAGNUCCI R. \& MARENGO J., 2009 - Present-day South American Climate. Palaeogeography Palaeoclimatology Palaeoecology, 281, 10.1016/j. palaeo.2007.10.032. 
JOMELLI V., FAVIER V., RABATEL A., BRUNSTEIN D., HOFFMANN G. \& FRANCOU B., 2009 - Fluctuations of glaciers in the tropical Andes over the last millennium and palaeoclimatic implications: A review. Palaeogeography Palaeoclimatology Palaeoecology, 281, doi: $10.1016 /$ j.palaeo.2008.10.033.

JOUZEL J., VIMEUX F., CAILLON N., DELAYGUE G., HOFFMANN G., MASSON-DELMOTE V. \& PARRENIN F., 2003 Temperature reconstruction from antarctic ice cores. Journal of Geophysical Research, 108 (D12), 4361-4372.

NOVELLO V. F., VUILLE M., CRUZ F. W., STRIKIS N. M., SAITO DE PAULA M., EDWARDS L., CHENG H., KARMANN I., JAQUETO P. F., TRINDADE R. I. F., HATMANN G. A. \& MOQUET J. S., 2016 - Centennial-scale solar forcing of the South American Monsoon System recorded in stalagmites. Nature Scientific reports, 6 (24762), doi:10.1038/srep24762.

RAMIREZ E., HOFFMANN G., TAUPIN J. D., FRANCOU B., RIBSTEIN P., CAILLON N., FERRON F. A., LANDAIS A., PETIT J-R., POUYAUD B., SCHOTTERER U., SIMOES J.C. \& STIEVENARD M., 2003 - A new Andean deep ice core from Nevado Illimani (6350 m), Bolivia. Earth and Planetary Sciences Letters, 212, 337-350.

THOMPSON L.G., MOSLEY-THOMPSON E., BOLZAN J.F. \& KOCI B.R., 1985 - A 1,500-year record of tropical precipitation in ice core from the Quelccaya ice cap, Peru. Science, 229, 971-973.

THOMPSON L.G., MOSLEY-THOMPSON E., DAVIS M. E., LIN P-N., HENDERSON K.A., COLE-DAI J., BOLZAN J.F. \&
LIU K.B., 1995 - Late glacial stage and Holocene tropical ice core records from Huascarán, Peru. Science, 269, 46-50.

THOMPSON L.G., DAVIS M. E., MOSLEY-THOMPSON E., SOWERS T., HENDERSON K.A., ZAGORODNOV V.S., LIN P-N., MIKHALENKO V.N., CAMPEN R.K., BOLZAN J.F., COLE-DAI J. \& FRANCOU B., 1998 - A 25,000-year tropical climate history from Bolivian ice cores. Science, 282, 1858-1864.

VIMEUX F., GALLAIRE R., BONY S., HOFFMANN G. \& CHIANG. J., 2005 - What are the climate controls on isotopic composition $(\delta \mathrm{D})$ of precipitation in Zongo Valley (Bolivia)? Implications for the Illimani ice core interpretation. Earth and Planetary Sciences Letters, 240, 205-220.

VIMEUX F., DE ANGELIS M., GINOT P., MAGAND O., POUYAUD B., CASASSA G., JOHNSEN S. \& FALOURD S., 2008 - A promising location in Patagonia for paleoclimate and environmental reconstructions revealed by a shallow firn core from Monte San Valentin (Northern Patagonia Icefield, Chile). Journal of Geophysical Research, 113, D16118, doi:10.1029/2007JD009502.

VIMEUX F., TREMOY G., RISI C. \& GALLAIRE R., 2011 - A strong control of the South American SeeSaw on the intra-seasonal variability of the isotopic composition of precipitation in the Bolivian Andes. Earth and Planetary Sciences Letters, 307, 47-58. 